\title{
Taxa Metabólica de Repouso de Ciclistas Estimada Por Equações e Obtida Por Calorimetria Indireta
}

\author{
Resting Metabolic Rate of Cyclists Estimated by Mathematical \\ Equations and Obtained by Indirect Calorimetry
}

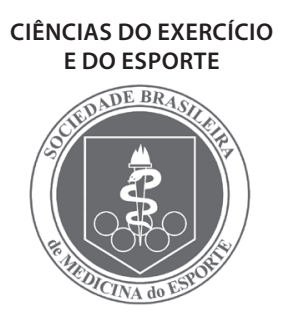

Artigo Original

\begin{abstract}
Paula Guedes Cocate Rita de Cássia Gonçalves Alfenas² Letícia Gonçalves Pereira ${ }^{3}$ João Carlos Bouzas Marins² Josefina Bressan²

Paulo Roberto Cecon ${ }^{2}$

1. Mestra em Ciências da Nutrição pela Universidade Federal de Viçosa, Viçosa-MG.

2. Professor(a) Dr.(a) da Universidade Federal de Viçosa, Viçosa-MG, 3. Bacharel em Nutrição pela Universidade Federal de Viçosa, Viçosa-MG.
\end{abstract}

\section{Endereço para correspondência:}

Paula Guedes Cocate.

Rua Liberato Antônio da Cunha, 110. 36780-000 - Astolfo Dutra, MG

(Brasil).

Tel.: (32) 3451-1712

Cel.: (32) 8412-6922.

E-mail: guedescocate@yahoo.com.br Instituição: Universidade Federal de Viçosa - MG (Brasil).

Submetido em: 26/02/2008 Versão final recebida em: 10/03/2009 Aceito em: 12/04/2009

\section{RESUMO}

A taxa metabólica de repouso (TMR) pode ser determinada por calorimetria indireta (CI). Porém, em função da praticidade, na prática clínica na maioria das vezes esta é estimada por equações de predição, as quais foram desenvolvidas em estudos envolvendo indivíduos não atletas. Apesar de alguns autores terem indicado que tais equações não estimam adequadamente a TMR, estas têm sido bastante utilizadas para calculá-la e prescrever dietas, inclusive para atletas. O objetivo deste estudo foi comparar a TMR determinada por $\mathrm{Cl}$ com a estimada pelas equações de Harris \& Benedict (HB), Schofield, FAO/WHO/UNU e Henry \& Rees (HR), em 15 homens ciclistas, de 24,4 \pm 3,68 anos, apresentando índice de massa corporal de 21,97 $\pm 1,46 \mathrm{~kg} / \mathrm{m}^{2}$ e VO 2 máx de 70,00 $\pm 5,32 \mathrm{~mL}(\mathrm{~kg} \cdot \mathrm{min})^{-1}$. Para comparar a TMR determinada por $\mathrm{Cl}$ e pelas equações, utilizou-se o tratamento estatístico testes $t$ de Student (variáveis com distribuição normal) e de Mann-Whitney (variáveis sem distribuição normal), considerando $p<0,05$. Além disso, foi realizada correlação de Pearson entre TMR e massa livre de gordura (MLG). Verificou-se que a TMR foi subestimada em 23,5\%, 16,7\%, 16,8\% e 16,9\%, respectivamente, quando se utilizaram as equações de HR, FAO/WHO/UNU, Schofield e HB, em relação à TMR obtida por Cl. A TMR se correlacionou com a MLG $(r=0,551, p=0,03)$. Os resultados confirmam que as equações avaliadas não são adequadas para estimar a TMR em atletas. Tais resultados podem ser explicados pela maior quantidade de MLG apresentada por atletas e possivelmente maior resposta dos receptores $\beta$-adrenérgicos aos estímulos do sistema nervoso simpático, resultando em TMR mais alta que em indivíduos sedentários. Diante disso, desenvolveu-se uma equação com as variáveis MLG e idade para melhor estimativa da TMR de ciclistas. Recomenda-se, no entanto, que a equação desenvolvida seja validada em estudo envolvendo um número maior de ciclistas, para que esta seja utilizada na prática clínica.

Palavras-chave: metabolismo de repouso, equações de predição, atletas.

\section{ABSTRACT}

The resting metabolic rate (RMR) can be determined by indirect calorimetry (IC). However, the clinical estimation of this parameter has been done using mathematical equations, which were developed in studies involving non-athletes. Although some authors have indicated that such equations do not estimate correctly the RMR, they have been constantly used to estimate such parameter and to prescribe diets, including for athletes. The objective of this study was to compare the RMR determined by IC with the ones estimated using the equations proposed by Harris \& Benedict (HB), Schofield, FAO/WHO/UNU and Henry \& Rees (HR), in 15 male cyclists, aged $24.4 \pm 3.68$ years, body mass index of $21.97 \pm 1.46 \mathrm{~kg} / \mathrm{m}^{2}$ and $\mathrm{VO}_{2}$ max of $70.00 \pm 5.32$ $\mathrm{mL}(\mathrm{kg} \cdot \mathrm{min})^{-1}$. Student's $t$ test (when data presented normal distribution) and Mann-Whitney (when data did not present normal distribution) were used to compare the RMR determined by IC and the ones estimated by the equations. Probability values below 0.05 were considered statistically significant. Pearson bivariate correlation coefficients were calculated to evaluate the relationship between RMR and fat free mass (FFM). It was verified that the RMR was significantly underestimated in 23.5\%, 16.7\%, 16.8\% and 16.9\%, respectively by the equations proposed by HR, FAO/WHO/UNU, Schofield and HB in comparison to RMR obtained by IC. RMR values were significantly correlated with FFM ( $r=0.551, p=0.03)$. These results confirm that the equations evaluated in this study are not appropriate to estimate the RMR of athletes. Such results can be explained by the fact that athletes have more FFM and possibly a greater $\beta$ - adrenergic receptors response to the sympathetic nervous system, which in turn leads to RMR higher than in sedentary people. Therefore, we created an equation considering the FFM and age variables to better estimate athletes' RMR. However, this equation should be validated in a study involving a greater number of cyclists before it can be clinically used.

Keywords: resting metabolism, estimative equations, athletes. 


\section{INTRODUÇÃO}

A taxa metabólica de repouso (TMR) corresponde ao consumo de energia por um indivíduo em repouso, desconsiderando os efeitos do consumo alimentar, da atividade física e do estresse mental ou fisiológico(1). Em indivíduos não atletas essa é responsável por 60 a 80\% do gasto energético diário ${ }^{(2)}$ e tem sido amplamente utilizada como base para se estimar a necessidade energética de uma dada população(3).

A TMR pode ser avaliada por calorimetria indireta, um método que determina as necessidades energéticas a partir do consumo de oxigênio e da produção de gás carbônico obtidos por meio do ar inspirado e expirado pelos pulmões ${ }^{(4)}$. A denominação indireta indica que a produção de energia é calculada a partir dos equivalentes calóricos do oxigênio consumido e do gás carbônico produzido. Assim, calcula-se a quantidade total de energia produzida, utilizando o oxigênio consumido para a oxidação dos substratos energéticos e o gás carbônico que é eliminado pela respiração(5).

Como na maioria das vezes não é possível mensurar a TMR por meio da calorimetria indireta em função do seu alto custo, foram desenvolvidas fórmulas que determinam essa taxa a partir dos valores de massa corporal, estatura e idade. Dentre as equações de predição deste parâmetro, a da FAO/WHO/UNU(6) e a de Schofield(7) foram desenvolvidas para determinar o gasto energético de indivíduos saudáveis. A equação de Henry \& Rees ${ }^{(8)}$ foi desenvolvida para pessoas saudáveis residentes em regiões tropicais. A equação de Harris \& Benedict ${ }^{(9)}$ foi desenvolvida para indivíduos enfermos, utilizando uma amostra de norte-americanos saudáveis ${ }^{(10)}$.

Para analisar a adequação das equações de predição da TMR, diversos pesquisadores têm comparado os valores obtidos utilizando tais equações com os obtidos por calorimetria indireta ${ }^{(3,11-15)}$. Dentre as equações anteriormente citadas, a de Harris \& Benedict ${ }^{(9)}$ tem sido a mais utilizada na prática clínica. Porém, alguns estudos têm mostrado que tal equação não representa adequadamente a TMR, por superestimá-la em indivíduos eutróficos e subestimá-la em obesos ${ }^{(11,12,14)}$. Os resultados de outros estudos têm demonstrado que as outras três equações (Schofield; FAO/WHO/UNU; Henry \& Rees) também não são precisas na estimativa da TMR em indivíduos saudáveis de ambos os sexos ${ }^{(3,13)}$.

Apesar disso, tais equações têm sido bastante utilizadas para estimar a TMR e a partir daí realizar a prescrição de dietas, inclusive para atletas. No entanto, pouco se sabe a respeito da aplicabilidade dessas equações para esportistas. Até a presente data, apenas um estudo nacional(16) foi conduzido para comparar a TMR de jogadores de futebol obtida por calorimetria indireta e pela equação de Harris \& Benedict. Os resultados do referido estudo indicam que tal equação superestima os valores da TMR também em atletas ${ }^{(16)}$. Não foi encontrado na literatura nenhum outro estudo que avaliou a adequação das demais equações existentes nesse sentido. Assim, o presente estudo foi realizado com o objetivo de avaliar a TMR de ciclistas do sexo masculino determinada por calorimetria indireta e estimada pelas equações de Harris \& Bene$\operatorname{dict}^{(9)}$, Schofield(7), FAO/WHO/UNU( ${ }^{(6)}$ e Henry \& Rees $^{(8)}$.

\section{MÉTODOS}

\section{Amostra}

A amostra foi composta de 15 atletas do sexo masculino, com idade entre 19 e 32 anos, apresentando peso estável (alteração < 3kg) nos últimos três meses e média de $\mathrm{VO}_{2}$ máx de $70,00 \pm 5,32 \mathrm{~mL}(\mathrm{~kg}$. $\mathrm{min}^{-1}$, não fumantes, não usuários de álcool ou de medicamentos que afetam a ingestão de alimentos ou o metabolismo energético. Todos os participantes assinaram um termo de consentimento livre e esclarecido, concordando em participar voluntariamente do estudo. Este foi aprovado pelo Comitê de Ética em Pesquisas com Seres Humanos da Universidade Federal de Viçosa-MG (parecer n 021/2006), atendendo às orientações da Resolução 196/96 do CNS, de 10/10/96, sobre experimentos com seres humanos.

\section{Desenho experimental}

A coleta de dados foi realizada no Laboratório de Metabolismo Energético e de Composição Corporal (Lamecc), da Universidade Federal de Viçosa-MG, em sala com baixa luminosidade, temperatura ambiente controlada (entre 24 e $26^{\circ} \mathrm{C}$ ) e umidade relativa do ar de $70-75 \%$. Cada voluntário compareceu ao laboratório em dois dias distintos com um intervalo de, no mínimo, uma semana entre os mesmos. Os voluntários foram orientados a se abster do consumo de café no dia anterior ao do teste, não consumir álcool nas 48 horas antecedentes, não realizar atividades físicas intensas $24 \mathrm{~h}$ antes dos testes e evitar a ingestão de água nas horas precedentes ao teste. Além disso, os atletas ficaram nas condições de jejum de no mínimo 12 horas e tiveram de seis a oito horas de sono na noite anterior em que o metabolismo energético foi avaliado.

No primeiro dia de coleta de cada voluntário, foram obtidas apenas as medidas antropométricas e de composição corporal, por um único avaliador com experiência em tais avaliações. A altura foi determinada utilizando-se um antropômetro vertical milimetrado, com extensão de $2 \mathrm{~m}$ e escala de $0,5 \mathrm{~cm}$. O peso, a massa corporal gorda e a massa corporal livre de gordura foram obtidos utilizando uma balança eletrônica digital da marca Tanita " modelo TBF-300A, com capacidade de 150kg, graduação de peso de 0,1 kg e graduação de gordura de 0,1\%. O percentual de gordura corporal foi medido utilizando tal balança, através do método da bioimpedância. Além disso, foi calculado o índice de massa corporal (IMC) ${ }^{(17)}$, relacionando o peso $(\mathrm{kg})$ e a altura (metros ao quadrado) apresentados.

Na segunda visita ao laboratório, os voluntários permaneceram em repouso por 30 minutos, deitados em uma cama. Em seguida, procedeuse à mensuração da TMR durante 30 minutos, com o avaliado deitado, sem se movimentar e acordado, utilizando o aparelho de calorimetria indireta (Deltatrac $\|^{\circledast}$, Datex, Finlândia). A TMR foi então obtida pela média dos valores encontrados durante os 30 minutos de avaliação nos dois dias de teste. A TMR assim obtida foi comparada com os valores de TMR (kcal em 24h) obtidos utilizando as equações de predição apresentadas no quadro 1.

Quadro 1. Equações de predição do gasto energético de repouso para homens adultos, as quais foram avaliadas no presente estudo.

\begin{tabular}{|c|c|}
\hline & Equações (kcal/24h) \\
\hline Harris \& Benedict ${ }^{(9)}$ & $\begin{array}{c}66,4730+13,7516(\mathrm{MC})+ \\
5,0033(\mathrm{E})-6,7550(\mathrm{I}) \\
\end{array}$ \\
\hline Schofield ${ }^{(7)}$ & $\begin{array}{l}18-30 \text { anos }[(0,0630(M C)+2,896)] \times 239 \\
30-60 \text { anos }[(0,0480(M C)+3,653)] \times 239\end{array}$ \\
\hline FAO/WHO/UNU(6) & $\begin{array}{l}18-30 \text { anos }[(0,0640(M C)+2,840)] \times 239 \\
30-60 \text { anos }[(0,0485(M C)+3,670)] \times 239\end{array}$ \\
\hline Henry \& Rees ${ }^{(8)}$ & $\begin{array}{l}18-30 \text { anos }[(0,0560(M C)+2,800)] \times 239 \\
30-60 \text { anos }[(0,0460(M C)+3,160)] \times 239\end{array}$ \\
\hline
\end{tabular}

Legenda: MC - massa corporal (kg), E - estatura (cm) e I - idade (anos)

Os percentuais de diferença entre os valores de TMR medidos por calorimetria indireta e os estimados pelas equações listadas acima foram calculados pela seguinte equação: [(TMR medida - TMR estimada)/TMR medida] $\times 100$.

Para a determinação do volume máximo de oxigênio $\left(\mathrm{VO}_{2}\right.$ máximo) e do nível de aptidão física dos voluntários ${ }^{(18)}$, os participantes foram submetidos ao teste máximo de Balke uma semana antes do início do experimento, utilizando um cicloergômetro eletromagnético, da marca Ergo Fit, modelo Ergo Cicle 167. Nesse teste, foram empregados estágios múltiplos, iniciando com carga de 50 watts, a qual foi aumentada a cada dois minutos, com magnitude de 50 watts, até o alcance do limite máximo ${ }^{(19)}$. A seguir, foi registrado o valor da última carga completada pelo voluntário em watts, bem como o valor do peso corporal avaliado antes da realização do teste, para o cálculo do $\mathrm{VO}_{2}$ máximo pela seguinte fórmula:

$$
\mathrm{VO}_{2 \max } \mathrm{mL}(\mathrm{kg} \cdot \mathrm{min})^{-1}=\frac{200+(12 \times \text { watts })}{\text { Peso }(\mathrm{kg})}
$$




\section{Análises estatísticas}

As análises estatísticas foram conduzidas para comparar os valores de TMR determinados por calorimetria indireta e os obtidos pelas equações de predição, utilizando o teste $t$ de Student para as variáveis com distribuição normal (Harris \& Benedict vs Deltatrac II) e o teste de MannWhitney para as variáveis que não apresentavam distribuição normal (FAO/WHO/UNU vs Deltatrac II, Schofield vs Deltatrac Il e Henry \& Rees vs Deltatrac (I). Além disso, foi realizada a correlação de Pearson para avaliar a correlação entre a TMR e a massa corporal livre de gordura e TMR com $\mathrm{VO}_{2}$ máx dos avaliados. As análises foram conduzidas utilizando o software SigmaStat 3.0(20), usando o nível de significância de $p<0,05$.

\section{RESULTADOS}

A tabela 1 apresenta as características da amostra estudada em valores de média ( \pm desvio padrão), mediana, valor máximo e valor mínimo. Já a tabela 2 apresenta os dados da TMR dos 15 ciclistas do sexo masculino obtida por calorimetria indireta e da calculada pelas equações de predição de Harris \& Benedict $^{(9)}$, Schofield ${ }^{(7)}$, FAO/WHO/ UNU(6), e Henry \& Rees $^{(8)}$.

Tabela 1. Idade e características antropométricas, de composição corporal e condicionamento físico da amostra estudada.

\begin{tabular}{l|c|c|c|c|c}
\hline \multicolumn{1}{c|}{ VARIÁVEL } & MÉDIA & DP & MEDIANA & MÍNIMO & MÁXIMO \\
\hline Idade (anos) & 24,40 & 3,68 & 25,00 & 19 & 32 \\
\hline Estatura (cm) & 174,73 & 4,91 & 1,74 & 168 & 185 \\
\hline $\mathrm{MC}(\mathrm{kg})$ & 67,11 & 5,52 & 67,30 & 57,00 & 76,80 \\
\hline $\mathrm{MLG}(\mathrm{kg})$ & 62,01 & 4,09 & 62,50 & 53,70 & 68,20 \\
\hline $\mathrm{MCG}(\mathrm{kg})$ & 5,30 & 2,10 & 4,80 & 1,80 & 10,30 \\
\hline $\mathrm{IMC}\left(\mathrm{kg} \cdot \mathrm{m}^{-2}\right)$ & 21,97 & 1,46 & 21,90 & 19,41 & 24,68 \\
\hline $\mathrm{GCT}(\%)^{1}$ & 7,72 & 2,49 & 7,10 & 3,10 & 13,40 \\
\hline $\mathrm{VO}_{2 \text { máx }} \mathrm{mL}(\mathrm{kg} \cdot \mathrm{min})^{-1}{ }^{*}$ & 70,00 & 5,32 & 71,42 & 57,80 & 77,32 \\
\hline$P^{-}$
\end{tabular}

DP - desvio padrăo, MC - massa corporal, MLG - massa livre de gordura, MCG - massa corporal gordurosa, IMC - índice de massa corporal, GCT- gordura corporal total

* Ausência de correlação com a taxa metabólica obtida por calorimetria indireta $(p=0,8)$

1 Percentual de gordura avaliado através do método da bioimpedância, por meio da balança digital Tanita", modelo TBF-300a

Tabela 2. Valores da taxa metabólica de repouso obtidos por calorimetria indireta e estimados pelas equações de predição.

\begin{tabular}{l|c|c|c|c|c}
\hline \multicolumn{1}{c|}{ VARIÁVEL } & MÉDIA & DP & MEDIANA & MÍNIMO & MÁXIMO \\
\hline $\begin{array}{l}\text { TMR por calorimetria } \\
\text { indireta (kcal/24h) }\end{array}$ & $2.051,33$ & 168,76 & $2.100,00$ & $1.730,00$ & $2.375,00$ \\
\hline $\begin{array}{l}\text { TMR por Harris \& } \\
\text { Benedict }{ }^{\mathrm{a}}(\mathrm{kcal} / 24 \mathrm{~h})\end{array}$ & $1.698,81$ & 95,14 & $1.685,29$ & $1.515,23$ & $1.872,57$ \\
\hline $\begin{array}{l}\text { TMR por Schofield } \\
\text { (kcal/24h) }\end{array}$ & $1.699,32$ & 84,53 & $1.703,18$ & $1.551,11$ & $1.849,24$ \\
\hline $\begin{array}{l}\text { TMR por FAO/WHO/ } \\
\text { UNUc (kcal/24h) }\end{array}$ & $1.701,93$ & 85,41 & $1.705,12$ & $1.550,63$ & $1.853,49$ \\
\hline $\begin{array}{l}\text { TMR por Henry \& } \\
\text { Rees }^{\mathrm{d}}(\mathrm{kcal} / 24 h)^{*}\end{array}$ & $1.562,46$ & 76,26 & $1.567,26$ & $1.432,09$ & $1.697,09$ \\
\hline PP $^{*}$
\end{tabular}

DP - desvio padrão, MC - massa crporal, TMR - taxa metabólica de repouso

* Valores de TMR determinados pelas equaçōes de prediçăo estatisticamente inferiores $(p<0,001)$ à TMR medida por calorimetria indireta

a $66,4730+13,7516(\mathrm{MC})+5,0033$ (estatura) - 6,7550 (idade)

${ }^{b} 18-30$ anos $[(0,0630(M C)+2,896)] \times 239$

$30-60$ anos $[(0,0480(M C)+3,653)] \times 239$

c $18-30$ anos $[(0,0640(M C)+2,840)] \times 239$

$30-60$ anos $[(0,0485(M C)+3,670)] \times 239$

d $18-30$ anos $[(0,0560(M C)+2,800)] \times 239$

$30-60$ anos $[(0,0460(M C)+3,160)] \times 239$

Verificou-se que a MLG se correlacionou $(r=0,55)$ estatisticamente $(p=0,03)$ com a TMR. A figura 1 representa a dispersão dos dados referentes à MLG e à TMR, demonstrando a ocorrência de relação crescente entre tais variáveis.

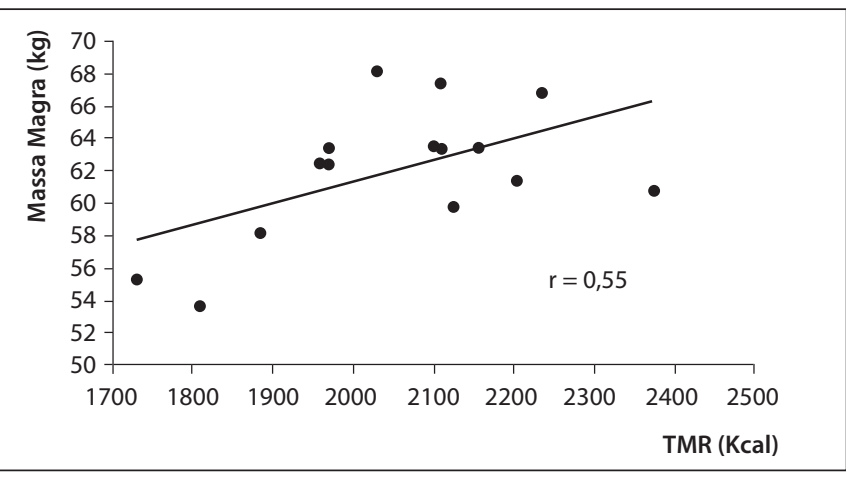

Figura 1

Dentre as equações de predição testadas no presente estudo, a que mais subestimou a TMR medida foi a de Henry \& Rees $(23,52 \pm 4,86 \%)$. O percentual de subestimação do valor da TMR determinada pelo Deltatrac e as estimadas pela equação da FAO/WHO/UNU (16,70 \pm 5,29\%), pela equação de Harris \& Benedict (16,90 $\pm 5,08 \%)$ e pela equação de Schofield (16,82 $\pm 5,28 \%)$ foi bastante semelhante (figura 2).

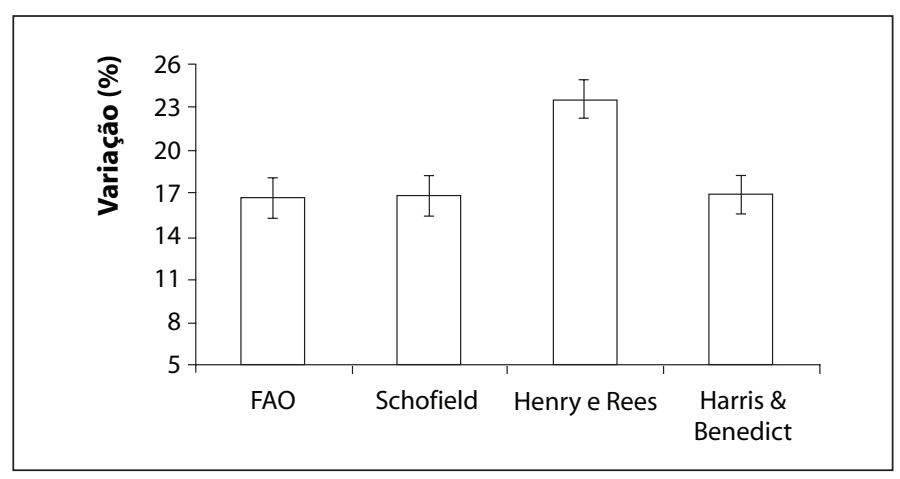

Figura 2

Diante da subestimação significante de todas as equações analisadas no presente estudo, foi desenvolvida uma nova equação para se estimar a TMR de indivíduos atletas. Para tal, foi ajustado um modelo matemático de regressão em função do teor de massa magra e da idade apresentada pelos participantes do atual estudo, obtendo a equação indicada a seguir:

$$
\mathrm{TMR}=-12878,2+485,846^{* *} \mathrm{MLG}-3,78467^{* *} \mathrm{MLG}^{2}-24,0092^{* *} \mid \mathrm{D}
$$

**: Significante ao nível de $1 \%$ de probabilidade, pelo teste $t$ de Student

Legenda: $M L G$ = Massa livre de gordura; ID = Idade.

A referida equação apresentou um coeficiente de determinação (R2) correspondente a 0,7147; assim, o modelo ajustado explica 71,47\% da variação total, sendo o restante $(28,53 \%)$ correspondente ao valor não explicado pelo modelo, ou seja, o erro.

\section{DISCUSSÃO}

No presente estudo, todas as equações avaliadas (Harris \& Benedict, FAO/WHO/UNU, Schofield e Henry \& Rees) subestimaram significantemente a taxa obtida por calorimetria indireta. Em contrapartida, os resultados de vários estudos ${ }^{(12,13,15)}$ têm apontado que as equações de predição atualmente disponíveis superestimaram a TMR em relação ao valor obtido por calorimetria indireta. Em um estudo ${ }^{(13)}$ envolvendo 60 mulheres brasileiras, com idade de 27,4 \pm 5,7 anos e IMC de 21,7 $\pm 2,7 \mathrm{~kg} /$ $\mathrm{m}^{2}$, residentes em Porto Alegre (RS), verificou-se que o valor referente ao gasto energético em repouso avaliado pelo calorímetro indireto foi superestimado em 17,1\% pela equação de Harris \& Benedict, em 13,5\% 
pela da FAO/WHO/UNU, 12,9\% pela fórmula de Schofield e 7,4\% pela equação de Henry \& Rees. Resultados semelhantes foram constatados por Cruz et al. (12) , em um estudo que envolveu a participação de 50 cariocas universitárias. A superestimação da TMR pelas equações de predição foi de 18,9\%, 12,5\% e 7,2\% para as equações de Harris \& Benedict, FAO/ WHO/UNU e Henry \& Rees, respectivamente. A divergência dos resultados da atual pesquisa com os demais estudos mencionados se deve ao fato de a população avaliada ter sido composta por atletas ciclistas, que apresentam características particulares em termos do nível de atividade física e composição corporal em relação a indivíduos sedentários.

As equações da FAO/WHO/UNU e Schofield foram criadas tomando-se como base os dados obtidos a partir de um mesmo grupo de indivíduos $^{(6)}$. A amostra utilizada no referido estudo ${ }^{(7)}$ foi constituída por $50 \%$ de soldados italianos, os quais eram ativos fisicamente e certamente apresentavam TMR superior à dos demais indivíduos menos ativos. No presente estudo, assim como no estudo de Wahrlich \& Anjos ${ }^{(13)}$, a TMR obtida utilizando as equações da FAO/WHO/UNU e Schofield foram semelhantes. Contudo, observou-se que tais equações também subestimaram os valores da TMR em relação àqueles obtidos por calorimetria indireta para os ciclistas.

Deve-se destacar que, por serem atletas, tais indivíduos apresentam mais massa muscular que indivíduos sedentários(21), levando ao aumento da TMR em relação a não atletas ${ }^{(15)}$. Faz-se importante ressaltar também que os participantes do presente estudo estavam habituados à elevada carga de treinamento físico. Segundo alguns autores, quanto mais alta a carga de treinamento, maior é o estímulo do sistema nervoso simpático e maior é a expressão dos receptores $\beta$-adrenérgicos, favorecendo o aumento da TMR ${ }^{(22)}$. Portanto, o aumento da TMR obtido no presente estudo pode ter ocorrido devido à influência do treinamento intenso e contínuo dos ciclistas.

A TMR de homens sedentários e ativos, com idade variando entre 18 e 60 anos, foi avaliada utilizando a equação da FAO/WHO/ONU e comparada com a obtida por meio de um calorímetro indireto ${ }^{(15)}$. Nos indivíduos sedentários de 18 a 30 anos e de 30 a 60 anos, a TMR mensurada por calorimetria indireta correspondeu a $1.480 \pm 160,19 \mathrm{kcal} /$ dia e $1.463 \pm$ $172,14 \mathrm{kcal} / \mathrm{dia}$, e a obtida pela equação da FAO/WHO/ONU foi de 1.790,8 $\pm 124,33 \mathrm{kcal} /$ dia e $1.711,9 \pm 62,16 \mathrm{kcal} /$ dia, respectivamente. Já para os homens fisicamente ativos, a TMR avaliada pela calorimetria foi de 1.723,8 $\pm 212,79 \mathrm{kcal} /$ dia e $1.656,9 \pm 157,8 \mathrm{kcal} /$ dia e a estimada foi de $1.790,8 \pm$ $148,2 \mathrm{kcal} /$ dia e 1.819,5 \pm 176,93kcal/dia para indivíduos de idade entre 18 e 30 anos e 30 e 60 anos, respectivamente. Os pesquisadores identificaram superestimação significante da TMR estabelecida pela equação proposta em nível internacional (FAO/WHO/ONU), tanto para indivíduos sedentários, quanto para os ativos. Apesar desses resultados, os autores apontam que a maior magnitude de diferença foi constatada em indivíduos sedentários. Ressalta-se que tal equação considera apenas o peso corporal, idade e gênero, excluindo outros fatores importantes, como a composição corporal.

Apesar de os participantes do estudo atual e daquele conduzido por Alfonzo-Gonzalez et al. ${ }^{(15)}$ terem apresentado o mesmo sexo e mesma faixa etária, no presente estudo foi obtida TMR maior utilizando a equação da FAO/WHO/ONU. Deve-se destacar, no entanto, que apesar de os autores daquele estudo ${ }^{(15)}$ terem indicado que os participantes praticavam exercícios físicos de alta intensidade regularmente, o nível de condicionamento físico dos mesmos não foi avaliado, dificultando assim melhor análise dos resultados obtidos ${ }^{(15)}$. O nível de condicionamento físico tem grande influência no aumento da TMR. Em geral, os indivíduos com alto nível de condicionamento físico apresentam mais massa magra e maior atividade do sistema nervoso simpático(21). Os atletas participantes do presente estudo apresentavam 7,4\% a mais de massa magra do que os participantes do estudo de Alfonzo-Gonzalez et al..(15).

Em outro estudo(16), a TMR de 50 atletas profissionais de futebol (homens) foi avaliada por calorimetria indireta, após 12 horas de jejum. O valor obtido foi comparado com o calculado pela equação de Harris \& Benedict. Os resultados indicaram que a equação superestimou (1.871 $\pm 126 \mathrm{kcal}$ por dia) os valores mensurados por calorimetria indireta (1.809 $\pm 126 \mathrm{kcal}$ por dia) dos atletas. Apesar de esse resultado divergir dos observados no presente estudo, em que foi constatada subestimação da TMR avaliada pela equação de Harris \& Benedict em relação à determinada por calorimetria indireta, os resultados obtidos no estudo de Garcia $^{(16)}$ estão em concordância com os dados obtidos nos estudos de Wahrlich \& Anjos ${ }^{(13)}$ e Cruz et al. ${ }^{(12)}$ com mulheres brasileiras.

Apesar de no estudo de Garcia(16) a composição corporal e condicionamento físico dos futebolistas não ter sido avaliada, sabe-se que os jogadores de futebol podem apresentar condicionamentos físicos bem distintos, em função das diferentes posições em que ocupam em campo. Em um estudo conduzido com jogadores profissionais, verificou-se que a média do percentual de gordura corporal dos mesmos era equivalente a 10,7 $\pm 1,4 \%{ }^{(22)}$. Esse valor pode ter favorecido a menor TMR obtida em relação ao alcançado pelos ciclistas participantes da pesquisa atual, os quais apresentavam menor percentual de gordura e consequentemente maior teor de massa livre de gordura.

Em relação à influência da massa livre de gordura na TMR, Speakman \& Selman ${ }^{(23)}$ relataram que a massa magra contribui com 50-70\% da TMR dependendo da variação interpessoal. Em concordância, Johnstone et al. ${ }^{(24)}$ verificaram que a massa livre de gordura explicou $62,3 \%$ da diferença nos valores da TMR de 150 pessoas de ambos os sexos, com idade entre 21 e 61 anos e IMC variando de 16,7 a 49,3 kg/m².

Seguindo a mesma linha de raciocínio, Illner et al. ${ }^{(25)}$ avaliaram a taxa metabólica de repouso de 13 mulheres e 13 homens eutróficos e saudáveis por meio do calorímetro indireto Deltatrac por uma hora e realizaram uma regressão linear dos valores assim obtidos com os da massa livre de gordura por bioimpedância. Os resultados indicaram elevada correlação $(r=0,92)$ entre ambas variáveis, revelando ainda que a massa livre de gordura explica 85\% da variação do taxa metabólica de repouso. Apesar de no presente estudo ter sido verificada correlação menor $(r=0,55)$, também foi constatada correlação positiva $(p=0,03)$ entre a TMR e massa livre de gordura dos atletas avaliados. Deve-se ressaltar, no entanto, que em outro estudo(26) também foi obtida correlação menor $(r=0,62)$ entre essas duas variáveis em atletas que participaram de um programa de treinamento para participação em uma maratona.

No estudo atual não foi identificada correlação significante entre o nível de condicionamento físico dos atletas ( $\mathrm{VO}_{2}$ máximo) e a TMR. Resultado semelhante foi obtido em outro estudo(27) que avaliou o $\mathrm{VO}_{2}$ máximo de mulheres em ergômetro de alta potência, utilizando um calorímetro indireto. Dentro desse contexto, Byrne \& Wilmore ${ }^{(21)}$, ao avaliar a diferença entre a TMR de mulheres treinadas e não treinadas, verificaram que as primeiras apresentavam maior TMR do que as outras. No entanto, tal diferença deixou de existir quando o valor da TMR era expresso em relação à quantidade de massa magra. Assim, esses autores verificaram por análise de regressão múltipla, usando a TMR como variável dependente e o VO 2 máximo e massa livre de gordura como preditor independente, que apenas a massa livre de gordura foi preditor significante da TMR expressa em $\mathrm{kcal} / \mathrm{dia}$. Assim, Byrne \& Wilmore ${ }^{(21)}$ justificaram a maior TMR das mulheres treinadas em função da maior quantidade de massa livre de gordura e da maior atividade do sistema nervoso simpático do que mulheres não treinadas. Dessa forma, a falta de correlação entre a TMR e $\mathrm{VO}_{2}$ máximo obtida no presente estudo envolvendo atletas suporta os resultados dos estudos de Smith et al.(27) e Byrne \& Wilmore ${ }^{(21)}$. Como os atletas possuem mais massa livre de gordura, os mesmos apresentam maior TMR do que indivíduos sedentários, justificando assim a subestimação da TMR calculada por meio das equações de predição, que normalmente consideram apenas o peso corporal para a determinação desse parâmetro.

No estudo atual, todos os atletas praticavam ciclismo por mais de três anos consecutivos, realizavam exercícios de intensidade moderada durante no mínimo 2,5 horas diárias e seis vezes por semana, ao longo dos treinos de endurance, além de realizarem durante uma hora diariamente exercício contrarresistência (musculação), também seis vezes por semana. Todos es- 
ses fatores podem ter interferido para o aumento da TMR. De acordo com Wilmore et al. ${ }^{(28)}$, o alto volume de endurance realizado durante anos tem um efeito positivo no aumento da TMR. Além disso, no estudo de Dolezal \& Potteiger ${ }^{(29)}$ foi verificado que a associação de treinamento de endurance e treinamento contrarresistência realizado por 30 homens jovens ativos durante 10 semanas promoveu aumento significante na TMR.

Dentro desse contexto, Tremblay et al. ${ }^{(30)}$ relataram que atletas de endurance apresentam maior TMR do que indivíduos não treinados. No entanto, após a infusão de propranolol (bloqueador de receptores $\beta$-adrenérgicos) nos atletas, essa diferença foi reduzida. Esse resultado sugere que a estimulação $\beta$-adrenérgica leva ao aumento da TMR e que os atletas altamente treinados apresentam maior atividade do sistema nervoso simpático (SNS) ou receptores $\beta$-adrenérgicos altamente regulados, promovendo o aumento da TMR. Para ilustrar melhor tal fato, Monroe et al. ${ }^{(31)}$ analisaram, em 29 jovens saudáveis (15 homens e 14 mulheres), o efeito do propranolol na TMR. Os resultados indicaram que o propranolol proporcionou redução significante da TMR $(1.555 \pm 26 \mathrm{kcal} /$ dia antes da infusão vs $1.484 \pm 29 \mathrm{kcal} /$ dia após a infusão), confirmando a influência positiva dos receptores $\beta$-adrenérgicos no aumento da TMR. Portanto, atletas podem apresentar receptores $\beta$-adrenérgicos tônicos mais ativos, os quais afetam o metabolismo energético de repouso, fazendo com que os valores de TMR sejam superiores aos observados em indivíduos não atletas.

Bell et al. ${ }^{(32)}$ também relataram que indivíduos treinados apresentam TMR superior à dos sedentários. No estudo conduzido por tais autores, 10 idosos altamente treinados foram submetidos a redução significante do nível de atividade física e da ingestão energética, por um período de cinco dias consecutivos. A TMR e a atividade do nervo simpático do músculo esquelético foram avaliadas antes e após o tratamento em condições normais e com a infusão de bloqueador dos receptores $\beta$-adrenérgicos (propranolol). Verificou-se que após a restrição calórica houve redução da TMR e da atividade do SNS. No entanto, observou-se que a infusão de propranolol reduziu a TMR apenas quando a restrição calórica não foi feita, indicando que a redução da atividade dos receptores $\beta$-adrenérgicos tônicos, envolvidos no controle do metabolismo energético de repouso, depende da restrição calórica. Os resultados desse estudo indicam que o elevado fluxo energético (alto consumo energético associado ao elevado gasto energético resultante da atividade física) em indivíduos altamente treinados leva ao aumento da TMR, em relação ao estado de baixo fluxo energético, que é característico de um público sedentário. Sendo assim, tais autores confirmam que indivíduos altamente treinados apresentam maior atividade do SNS, maior sensibilidade dos receptores

\section{REFERÊNCIAS BIBLIOGRÁFICAS}

1. Clark HD, Hoffer $\sqcup$. Reappraisal of the resting metabolic rate of normal young men. Am J Clin Nutr. 1991;53:21-6.

2. Pelt REV, Dinneno FA, Seals DR, Jones PP. Age-related decline in RMR in physically active men: relation to exercise volume and energy intake. Am J Physiol Endocrinol Metab. 2001;281:633-9.

3. Ismail MN, Ng KK, Chee SS, Roslee R, Zawiah H. Predictive equations for the estimation of basal metabolic rate in Malaysian adults. Mal J Nutr. 1998:4:81-90.

4. Diener, JRC. Artigo de Revisão: Calorimetria Indireta. Rev Ass Med Brasil. 1997;43:245-53.

5. Schneider P, Meyer F. As equações de predição da taxa metabólica basal são apropriadas para adolescentes com sobrepeso e obesidade? Rev Bras Med Esporte. 2005;11:193-6.

6. FAO/WHO/UNU. Energy and protein requirements. Geneva: World Health Organization,1985. [WHO Technical Report Series, 724

7. Schofield WN. Predicting basal metabolic rate, new standards and review previous work. Hum Nutr Clin Nutr. 1995;39C:5-41 8. Henry CJK, Rees DG. New predictive equations for the estimation of basal metabolic rate in tropical peoples. Eur J Clin Nutr. 1991;45:177-85.

9. Harris JA, Benedict FG. A biometric study of basal metabolism in man. Boston: Carnegie Institution of Washington, 1919 10. Wahrlich V, Anjos LA. Aspectos históricos e metabólicos da medição e estimativa da taxa metabólica basal: uma revisão de literatura. Cad Saúde Pública. 2001a;17:801-17.

11. Daly JM, Heymsfield SB, Head CA, Harvey LP, Nixon DW, Katzeff H, Grossman GD. Human energy requirements: overestimation by widely used prediction equation. Am J Clin Nutr. 1985;42:1170-4

12. Cruz CM, Silva AF, Anjos LA. A taxa metabólica basal é superestimada pelas equações preditivas em universitárias do Rio de Janeiro, Brasil. Arch Latinoam Nutr. 1999;49:232-7.

13. Wahrlich V, Anjos LA. Validação de equaçōes de predição da taxa metabólica basal em mulheres residentes em Porto Alegre-RS, Brasil. Rev Saúde Pública. 2001b;35:39-45.

14. Nonino CB. Calorimetria indireta x Harris Benedict: Determinação, validação e comparação para o cálculo da taxa metabólica de repouso em obesas grau III [dissertação]. São Paulo (SP): Faculdade de Medicina de Ribeirão Preto da Universidade de Säo Paulo; 2002.

15. Alfonzo-Gonzalez G, Doucet E, Almerás N, Bouchard C, Tremblay A. Estimation of daily energy needs with the FAO/WHO/ONU 1985 procedures in adults: comparison to whole-body calorimetry measurements. Eur J App Physiol. 2004;58:1125-31.

16. Garcia ES. Taxa metabólica basal de jogadores profissionais de futebol. Rev Bras Educ Fis Esp. 2006;20:82-4.

$\beta$-adrenérgicos e maior TMR que indivíduos sedentários, justificando tais mecanismos fisiológicos em decorrência do alto fluxo energético que o grupo de indivíduos altamente ativos apresenta.

A partir dessas informações, é possível inferir que a TMR de indivíduos ativos, sobretudo de atletas que têm elevada carga de treinamento físico diário, é superior à TMR de indivíduos sedentários, uma vez que indivíduos ativos fisicamente apresentam mais massa muscular e maior atividade do SNS. Portanto, os valores de TMR calculados a partir das equações de predição até então propostas por outros autores podem ter sido subestimados em decorrência dessas características específicas apresentadas pelos participantes do presente estudo. Desse modo, foi desenvolvida no presente estudo uma nova equação para melhor estimar a TMR de indivíduos atletas, considerando a massa magra e a idade apresentada pelos mesmos. Tal equação tem capacidade de explicar 71,27\% da TMR de indivíduos com a mesma faixa etária e mesma faixa de massa muscular dos participantes do presente estudo, tendo esta, portanto, um erro de apenas 28,53\%. Considera-se ainda que a referida equação seja mais adequada na estimativa da TMR de ciclistas do que as demais equações de predição aqui avaliadas, porém, cabe ressaltar que tal equação é limitada para indivíduos que apresentam características semelhantes quanto à idade (19 a 32 anos) e massa corporal magra $(53,7$ e 68,2kg) dos ciclistas que participaram da atual pesquisa.

\section{CONCLUSÃO}

Os resultados obtidos neste estudo evidenciaram que as equações de predição disponíveis atualmente não são adequadas para estimar a TMR em atletas, já que esse parâmetro depende da quantidade de massa livre de gordura apresentada pelos mesmos. Tais equações foram desenvolvidas em estudos envolvendo indivíduos não atletas saudáveis. Assim, recomenda-se que a estimativa da TMR de atletas seja feita utilizando equações que considerem o teor de massa livre de gordura. No presente estudo foi desenvolvida uma nova equação, considerando essa variável com o intuito de proporcionar uma estimativa mais adequada da TMR de ciclistas. Recomenda-se, no entanto, que novos estudos envolvendo um número maior de atletas sejam conduzidos para validar tal equação, para que a mesma possa ser recomendada e utilizada na prática por nutricionistas, médicos e educadores físicos.

\section{AGRADECIMENTO}

Agradecemos à Fapemig (Fundação de Amparo à Pesquisa do Estado de Minas Gerais) pela concessão do auxílio financeiro (processo 1.420/06) para a condução do presente estudo.
17. Bray GA, Gray DS. Obesity I: Phathogenesis. Western J Med. 1988;149:429-41.

18. Cooper KH. O programa aeróbico para o bem estar total. Rio de Janeiro: Nórtica, 1982.

19. Marins J, Giannichi R. Avaliação e prescrição de atividade física. 3 rd rev. ed. Rio de Janeiro: Shape, 2003. 20. SPSS Inc. Sigma Stat for Windows [computer program]. Version 3.0. 2003

21. Byrne HK, Wilmore JH. The relationship of mode and intensity of training on resting metabolic rate in woman. Int J Sport Nutr Exerc Metab. 2001;11:1-14.

22. Campeiz JM, Oliveira PR de, Maia GBM. Análise de variáveis aeróbias e antropométricas de futebolistas profissionais, juniores e juvenis. Conexões. 2004;2:1-19.

23. Speakman JR, Selman C. Physical activity and resting metabolic rate. Proc Nutr Soc. 2003;62:621-34.

24. Johnstone AM, Murison SD, Duncan JS, Rance KA, Speakman JR. Factors influencing variation in basal metabolic rate include fat-free mass, fat mass, age, and circulating thyroxine but not sex, circulating leptin, or triiodothyronina Am J Clin Nutr. 2005;82:941-8.

25. Illner K, Brinkmann G, Heller M, Bosy-Westphal A, Muller MJ. Metabolically active components of fat free mass and resting energy expenditure in nonobeses adults. Am J Physiol Endocrinol Metab. 2000;278:E308-15.

26. Westerterp KR, Meijer GA, Schoffelen P, Janssen EM. Body mass, body composition and sleeping metabolic rate before, during and after endurance training. Eur J Appl Physiol Occup Physiol. 1994;69:203-8.

27. Smith DA, Dollman J, Withers T, Brinkman M, Keeves JP, Clark DG. Relationship between maximum aerobic power and resting metabolic rate in young adult women. J Appl Physiol. 1997;82:156-63.

28. Wilmore JH, Stanforter PR, Hudspeth LA, Gagnon J, Daw EW, Leon AS, et al. Alterations in resting metabolic rate as a consequence of 20 wk of endurance training: the HERITAGE Family Study. Am J Clin Nutr. 1998;68:66-71.

29. Dolezal BA, Potteiger JA. Concurrent resistance and endurance training influence basal metabolic rate in nondieting individuals. J Appl Physiol. 1998;85:695-700.

30. Tremblay A, Coveney S, Despres JP, Nadeau A, Prud'homme D. Increased resting metabolic rate and lipid oxidation in exercise-trainned individuals: evidence for a role of beta-adrenergic stimulation. Can J Physiol Pharmacol. 1992;70:1342-7.

31. Monroe MB, Seals DR, Shapiro LF, Bell C, Johnson D, Jones PP. Direct evidence for tonic sympathetic support of resting metabolic rate in healthy adult humans. Am J Physiol Endocrinol Metab. 2001;280:E740-4.

32. Bell C, Day DS, Jones PP, Christou DD, Petitt DS, Osterberg K, et al. High energy flux mediates the tonically augmented badrenergic support of resting metabolic rate in habitual exercising older adults. J Clin Endocrinol Metab. 2004;89:3573-8. 